\title{
Pensamiento computacional, Robótica y Programación en educación
}

\author{
Computational Thinking, Robotics and Coding in Education
}

\author{
María del Mar Sánchez-Vera \\ Universidad de Murcia (España) \\ mmarsanchez@um.es \\ Juan González-Martínez \\ Universitat de Girona (España) \\ juan.gonzalez@udg.edu
}

\begin{abstract}
El debate en torno al pensamiento computacional (en adelante PC) y sus posibilidades en los diferentes contextos educativos no es novedoso. Ya en 1980, Papert hablaba en Mindstorms de la importancia de integrar el PC en el día a día educativo (Papert, 1980). En concreto, a partir de su teoría de aprendizaje construccionista, abría la puerta a considerar el robot como un objeto con el que pensar, y el lenguaje de programación como un medio con el que expresarnos y dar forma a nuestras ideas.

Tras unas décadas en las que el concepto siguió vigente, pero no de modo generalizado, el PC resurgió internacionalmente cuando en 2006 Jeannette Wing destacó su importancia como estrategia eficaz para la resolución de problemas, por medio de la réplica de estrategias tomadas de la computación (Wing, 2006); y puso en el foco la idea de que utilizar estas estrategias pueden ayudar a desarrollar destrezas aplicables a muchas otras situaciones. El PC, por tanto, no es solo una alfabetización necesaria para quienes deban dedicarse profesionalmente a la programación (o al ámbito científico-tecnológico en general), sino que es transferible a multitud de contextos personales y, por tanto, necesario por útil para toda la ciudadanía.
\end{abstract}

Esta nueva perspectiva, unida al rápido desarrollo de herramientas de programación y robótica, cada vez más intuitivas y especialmente pensadas para su uso educativo o para la población general, ha catapultado el debate en torno a las potencialidades del PC a una fuerte actualidad en todos los niveles y en todos los contextos educativos, desde la Educación Infantil hasta la formación universitaria.

Con todo, el PC como tal no conoce aún una definición que reúna consenso general en la comunidad científica y educativa internacional; ni están claras las imbricaciones entre él y la robótica o la programación. Por otro lado, la investigación al respecto es incipiente y, en consecuencia, en ocasiones poco sistemática. Y la práctica educativa se impone en la realidad, urgida por la necesidad de dar respuesta a las necesidades y a las demandas de la sociedad e impelida también por los desarrollos tecnológicos comerciales, que toman de facto parte de las decisiones que el ámbito educativo deja sin respuesta. Para acabar de concretar el marco, la legislación educativa intenta reflejar esa velocidad de la práctica, pero sin el colchón de los avances de la investigación. Eso refleja en buena medida los distintos enfoques que existen de cara a la incorporación del PC en las aulas: uno más técnico y ligado a la programación, que considera que el PC se equipara básicamente a saber programar y aplicar líneas de código; y otro enfoque más abierto y ligado a perspectivas más amplias, que considera que el PC es algo transversal y relacionado con la alfabetización o competencia digital.

Las múltiples vías de pensamiento que genera esta rápida eclosión, como decíamos, son también de índole ética: la aplicación del PC al mundo educativo va de la mano del desarrollo tecnológico de dispositivos que permiten aplicarlo de modo más visual, o más atractivo y lúdico. 
Y así, aunque el desarrollo de esta alfabetización puede darse sin ningún componente tecnológico asociado (el llamado PC unplugged o desenchufado), ya desde las primeras aproximaciones conceptuales de Papert son indudables el protagonismo del robot (como dispositivo) y del lenguaje de programación (como vehículo del pensamiento abstracto), lo cual abre la puerta a multitud de iniciativas que explotan ese filón también desde el punto de vista comercial (con propuestas que no siempre están al alcance de todos los públicos, y que cercenan esa idea general de universalización del PC como alfabetización con forzosa vocación general). Las aristas del aterrizaje del PC (y de la robótica y de la programación) en las aulas, como se ve, también se plantean en términos éticos ligados al consumo y a la presión de las empresas tecnológicas.

En España, por partir de nuestro contexto más inmediato, y según el informe del INTEF (2018), la integración del PC se está produciendo principalmente en la Educación Primaria y Educación Secundaria. Y, en cuanto a cómo se concreta su presencia en las aulas, resulta interesante también conocer que, de forma general, en la etapa de Infantil se trabaja de forma transversal en la acción docente, si bien conforme aumentamos de nivel educativo hasta Secundaria, es más frecuente encontrar una asignatura específica de programación o robótica.

Ante esta situación, y según este mismo informe, muchos maestros se forman de manera autónoma para dar respuesta a cómo integrar la robótica en sus clases. En la red, muchos encuentran un lugar adecuado para intercambiar experiencias y autoformarse de modo informal. Y es eso lo que da pie, por ejemplo, a destacadas iniciativas como Robótica por la lgualdad (https://roboticaporlaigualdad.blogspot.com/=), un movimiento en red de profesorado interesado en integrar el PC en sus aulas, basándose en la colaboración entre centros y en el préstamo de robots, para que todos los alumnos tengan la oportunidad de aprender a programar con tecnología. Y es en ese sentido en el que hablábamos antes de los condicionantes económicos: aunque cada vez hay opciones más asequibles, la dotación de robótica en las aulas conlleva un gasto que no todos los centros pueden afrontar. Por ello, este tipo de experiencias educativas son realmente interesantes, ya que aprovechan el potencial de la tecnología no solo para el aprendizaje sino también para el desarrollo de la igualdad social.

Aterrizando ya en el ámbito específico de la tecnología educativa, desde donde abordamos el PC en la Revista Interuniversitaria de Investigación en Tecnología Educativa, el PC nos presenta un panorama realmente interesante. Por un lado, la incorporación de los robots como panacea educativa nos tiene que llevar a rescatar las bases fundamentales de la tecnología educativa en el marco de la didáctica: no existe el supermedio. $Y$, aunque considerásemos que el robot puede convertirse un instrumento estrella, con un protagonismo pedagógico hasta ahora desconocido, si no está integrado de manera adecuada en la estrategia didáctica docente, nos perderemos todas las potencialidades de esa herramienta, y volverá a ser otro recurso superado en el futuro por otra tecnología nueva diferente. A nuestro entender, sin duda, esto es lo que nos catapulta a fortalecer el discurso del code to learn y, por tanto, a centrarnos en el desarrollo del PC desde su perspectiva de nueva alfabetización del siglo XXI, ya que puede permitir ayudarnos a establecer una nueva relación con la tecnología, de tal forma que no seamos solo consumidores, sino productores de la misma.

En este sentido, retomemos los dos elementos que acabamos de añadir a esta reflexión, y que, si bien están relacionados con el PC, no son una misma cuestión, como son la programación y la robótica. A veces se considera que por enseñar a programar automáticamente estaremos desarrollando el PC de las personas. Sin embargo, sentar al alumnado a escribir líneas de código sin entender la lógica de trabajo que hay detrás probablemente sirva de poco. De nuevo, la tecnología no puede quedarse en lo meramente técnico, sino que es necesario abordar a nivel metodológico el potencial que tiene usar la programación y la robótica, de manera coherente en el currículo educativo. Del mismo modo, resulta muy interesante también valorar las posibilidades didácticas del PC desenchufado y la programación de videojuegos y aplicaciones, que bien integrados pueden ayudarnos a favorecer su desarrollo.

Como consecuencia de todo ello, este monográfico para la revista RIITE se concibe como un intento práctico de contribuir a la reflexión en torno al concepto de pensamiento computacional, para realizar algunas aportaciones relevantes que se pueden hacer desde la Tecnología Educativa en torno al PC y cómo trabajarlo en contextos educativos y conocer 
diversas perspectivas en torno al mismo. Y, para ello, contamos con seis aportaciones en forma de artículo científico, y una entrevista que a buen seguro nos ayudarán a aportar un poco de luz al respecto.

En referencia a la entrevista, en este número Marta Reina, jefa de servicio del área de formación y competencia digital en el Instituto Nacional de Tecnología Educativa (INTEF), nos presenta un análisis sobre cómo el PC puede ayudar a que el alumnado desarrolle habilidades de resolución de problemas, al descomponerlo en otros más pequeños y establecer secuencias de pasos ordenadas. También presenta una interesante visión acerca de cómo se ha de integrar en el currículo educativo y qué papel han de desempeñar los distintos agentes (profesorado, universidades, empresas tecnológicas) en torno a ello.

En cuanto a los artículos, Jesús Moreno-León, Gregorio Robles, Marcos Román-González y Juan David Rodríquez abordan en Not the same: a text network analysis on computational thinking definitions to study its relationship with computer programming uno de los aspectos teóricos relevantes en cuanto al PC, como es la aparente dispersión conceptual que se deriva de no contar con una definición clara y comúnmente aceptada en el ámbito de conocimiento. Su análisis de red de texto de las principales conceptualizaciones vigentes en las referencias bibliográficas más citadas revela que los nodos principales son compartidos y que, por tanto, a pesar de las divergencias en la priorización o en las ramificaciones conceptuales, el tronco conceptual es común en todas ellas.

A continuación, Pensamiento computacional en el aula: el desafío en los sistemas educativos de Latinoamérica es el siguiente artículo que podemos encontrar en el monográfico. Es un trabajo de Enríque Arturo Vázquez, Johanna Bottamedi y María Luz Brizuela. Estos autores realizan un buen trabajo de revisión y análisis de cómo se ha integrado el PC en Argentina, Urugay, Chile y México, en el marco de las políticas públicas de enseñanza, en dónde se pueden percibir también distintas perspectivas en torno a las cómo integrar el PC en contextos escolares.

Por su parte, Hamilton Omar Pérez, Alex Álvarez-Zurita y Carlos Roberto Guevara analizan en Dominio de habilidades del pensamiento computacional en los estudiantes del Instituto Tecnológico Superior Sucre de Quito - Ecuador la relación que se establece entre diferentes elementos del PC en su desarrollo en alumnado inicial de los estudios universitarios técnicos a fin de comprobar la heterogeneidad de perfiles de ingreso en relación con la formación previa y su incidencia posterior en los estudios.

En cuarto lugar, el artículo de Eneko Tejada, Ainara Romero, Aranzazu López y Naiara Bilbao, titulado Análisis y creación de máquinas virtuales cognitivas: percepción de aprendizaje útil en el alumnado universitario, nos presenta un novedoso enfoque de desarrollo del PC trabajado con el alumnado de la Facultad de Educación de la Universidad del País Vasco, a través de máquinas virtuales cognitivas, que permiten definir un grupo de objetos y algoritmos que posibilitan la resolución de problemas complejos.

A continuación, Salomé Recio, en su artículo Experiencias robóticas en Infantil nos evidencia el gran potencial que tiene la robótica en este nivel educativo, y cómo se puede contribuir al desarrollo del pensamiento computacional en estos primeros cursos escolares, a partir de experiencias de aprendizaje significativo y ejemplos prácticos de aula.

Y, finalmente, Carina Soledad González, en su artículo Estrategias para la enseñanza del pensamiento computacional y uso efectivo de tecnologías en educación infantil: una propuesta inclusiva, describe los principios pedagógicos y las principales estrategias de enseñanza del PC y la programación para la educación partiendo de una perspectiva inclusiva, desde la que presenta una propuesta educativa para la enseñanza-aprendizaje de la programación y el PC para la Educación Infantil.

Para terminar, el monográfico cierra con una reseña off-topic dedicada a una de las estrategias metodológicas de interés en la actualidad, el aula invertida. Esta reseña, realizada por Salvador Montaner, presenta el libro publicado por Raúl Santiago y Jon Bergmann titulado Aprender al revés. Flipped Learning 3.0 y metodologías activas en el aula. En él, se desgranan elementos sustanciales de la clase invertida en particular y de las metodologías activas en general, como los roles del profesorado y del alumnado o las pautas que se recomiendan para 
su aplicación. Asimismo, también se sugieren algunas buenas prácticas y algunas evidencias de investigación sobre las vías de oportunidad que esta estrategia puede ofrecer al profesorado.

\section{REFERENCIAS BIBLIOGRÁFICAS}

INTEF (2018). Programación, robótica y Pensamiento Computacional en el aula. Situación en España, enero 2018. Madrid, Ministerio de Educación, Instituto Nacional de Tecnologías Educativas y de Formación del Profesorado. Disponible en http://code.intef.es/wpcontent/uploads/2017/09/Pensamiento-Computacional-Fase-1-Informe-sobre-lasituaci\%C3\%B3n-en-Espa\%C3\%B1a.pdf

Papert, S. (1980). Mindstorms. Children, Computers and Powerful Ideas, New York (EE. UU.), Basic Ideas.

Wing, J. M. (2006). Computational Thinking. Communications of the ACM, 49(3), 33-35. https://doi.org/10.1145/1118178.1118215

\section{INFORMACIÓN SOBRE LOS AUTORES}

\section{María del Mar Sánchez-Vera \\ Universidad de Murcia}

Profesora titular del Departamento de Didáctica y Organización Escolar de la Facultad de Educación de la Universidad de Murcia y miembro del Grupo de Investigación de Tecnología Educativa (GITE) y el Grupo de Innovación Docente en Tecnología Educativa. Ha sido profesora visitante en la Universidad de Cambridge, la Universidad John Moore de Liverpool y la Universidad de Southampton. Sus principales intereses de investigación se relacionan con el análisis y el desarrollo de recursos y actividades con TIC en la educación, el estudio de las estrategias metodológicas y los entornos virtuales para la formación en línea, y la formación docente en el marco de la Tecnología Educativa.

ORCID: https://orcid.org/0000-0003-4179-6570

\section{Juan González-Martínez}

Universitat de Girona

Profesor Agregado Serra Húnter del Departament de Pedagogia de la Universitat de Girona. Doctor en Tecnología Educativa por la Universitat Rovira i Virgili (Tarragona, España) y en Lengua Española por la Universidad Nacional de Educación a Distancia (Madrid, España). Miembro del grupo de investigación consolidado UdiGitalEdu y de los grupos de innovación docente Transmèdia i Educació i Narratives Digitals en Educació Social de la Universitat de Girona. Profesor Visitante del programa de Doctorado en Tecnología Educativa de la Universitat Rovira i Virgili y de la Universidad Autónoma de Querétaro (México). Sus principales intereses de investigación se centran en la competencia digital de los estudiantes y en la competencia digital docente, así como en el transmedia y su uso educativo.

ORCID: https://orcid.org/0000-0002-9175-6369

Los textos publicados en esta revista están sujetos a una licencia de Reconocimiento 4.0 España de Creative Commons. Puede copiarlos, distribuirlos, comunicarlos públicamente y hacer obras derivadas siempre que reconozca los créditos de las obras (autoría, nombre de la revista, institución editora) de la manera especificada por los autores o por la revista. La licencia completa se puede consultar en:Licencia Creative Commons Atribución-NoComercial-Compartir por igual 4.0 Internacional. 